The University of Southern Mississippi

The Aquila Digital Community

Faculty Publications

8-21-2009

\title{
On Immunotherapies and Cancer Vaccination Protocols: A Mathematical Modelling Approach
}

\author{
Badal Joshi \\ Ohio State University \\ Xueying Wang \\ Ohio State University \\ Sayanti Banerjee \\ Ohio State University \\ Haiyan Y. Tian \\ University of Southern Mississippi, Haiyan.Tian@usm.edu \\ Anastasios Matzavinos \\ lowa State University, tasos@iastate.edu
}

See next page for additional authors

Follow this and additional works at: https://aquila.usm.edu/fac_pubs

Part of the Physical Sciences and Mathematics Commons

\section{Recommended Citation}

Joshi, B., Wang, X., Banerjee, S., Tian, H. Y., Matzavinos, A., Chaplain, M. A. (2009). On Immunotherapies and Cancer Vaccination Protocols: A Mathematical Modelling Approach. Journal of Theoretical Biology, 259(4), 820-827.

Available at: https://aquila.usm.edu/fac_pubs/1150

This Article is brought to you for free and open access by The Aquila Digital Community. It has been accepted for inclusion in Faculty Publications by an authorized administrator of The Aquila Digital Community. For more information, please contact Joshua.Cromwell@usm.edu. 


\section{Authors}

Badal Joshi, Xueying Wang, Sayanti Banerjee, Haiyan Y. Tian, Anastasios Matzavinos, and Mark A.J. Chaplain 


\title{
On immunotherapies and cancer vaccination protocols: a mathematical modelling approach
}

\author{
Badal Joshi ${ }^{\mathrm{a}}$, Xueying Wang ${ }^{\mathrm{a}}$, Sayanti Banerjee ${ }^{\mathrm{a}}$, Haiyan Tian ${ }^{\mathrm{b}}$, \\ Anastasios Matzavinos ${ }^{\mathrm{a}, *}$ and Mark A.J. Chaplain ${ }^{\mathrm{c}}$ \\ a Department of Mathematics, The Ohio State University, Columbus, OH 43210, USA \\ b Department of Mathematics, University of Southern Mississippi, Hattiesburg, MS 39406, USA \\ ${ }^{c}$ Department of Mathematics, University of Dundee, Dundee, DD1 4HN, UK
}

June 9, 2008

\begin{abstract}
In this paper we develop a new mathematical model of immunotherapy and cancer vaccination, focusing on the role of antigen presentation and costimulatory signaling pathways in cancer immunology. We investigate the effect of different cancer vaccination protocols on the well-documented phenomena of cancer dormancy and recurrence, and we provide a possible explanation of why adoptive immunotherapy protocols can sometimes promote tumor growth instead of inhibiting it (a phenomenon called immunostimulation), as opposed to active vaccination protocols based on tumor-antigen pulsed dendritic cells. Significantly, our simulations suggest that elevated numbers of professional antigen presenting cells correlate well with prolonged time periods of cancer dormancy.
\end{abstract}

\section{Introduction}

Cancer still remains one of the most difficult diseases to treat clinically and is one of the main causes of mortality in developed western societies. The mortality statistics for the United Kingdom for the year 2005 show that 153,491 people were registered as dying from a malignant neoplasm 11 This figure represents $26 \%$ of all causes of death in the UK for 2005 , and similar statistics hold for the United States (Ries et al., 2007).

Great effort and resources are devoted to cancer research and our understanding of cancer biology is constantly expanding. However, the overall efficiency of our current therapeutic approaches remains rather poor. Current patient therapies for the treatment of cancer include surgery (i.e. removal of the tumour), chemotherapy (administration of anti-cancer drugs) and radiotherapy (treatment with X-rays). Of course surgery is appropriate only for solid tumours. Although there have been great advances in patient care and treatment

\footnotetext{
*E-mail address: tasos@math.ohio-state.edu

${ }^{1}$ Source: Cancer Research UK
}

over the past few decades with refinement of anticancer drugs and medical equipment, unfortunately chemotherapy and radiotherapy both still carry major side-effects for individual patients. This is mainly due to the severe effects that these treatments have on normal, healthy proliferating cells in the patients. As a result, the treatment of cancers itself causes significant morbidity and mortality.

Given these facts any design of new therapeutic approaches is of great interest and one such new approach is to treat cancer using key components of the immune system, the body's natural defence mechanism (Abbas et al., 2007). In recent years there has been much biological, immunological and experimental interest in trying to develop what may be termed "immunotherapies" for cancers. One major advantage that some form of effective immunotherapy treatment would have over conventional anticancer treatment would be the fact that cells and other components of the immune system would be far more specific and localised in their actions, targetting cancer cells alone and leaving the vast majority of other healthy cells of the body untouched (Parmiani \& Lotze, 2002). 
As part of a deeper understanding of cancer therapy the role of quantitative and predictive mathematical modelling is becoming increasingly appreciated by experimentalists and clinicians, and in recent years several papers have begun to investigate the various aspects of the immune system response to cancer from a mathematical perspective. The development of mathematical models which reflect several spatial and temporal aspects of tumour immunology can be regarded as the first step towards an effective computational approach in investigating the conditions under which tumour recurrence takes place and in optimizing existing immunotherapy protocols. Key papers in this area include (Ambrosi et al., 2002; Arlotti et al., 2002; De Angelis et al., 2003; Bellomo et al., 2003; Bellomo et al., 1999; Bellomo \& Preziosi, 2000), which focus on the modelling of tumour progression and immune competition by generalized kinetic (Boltzmann) models and (Owen \& Sherratt, 1997; Owen \& Sherratt, 1998; Owen \& Sherratt, 1999; Sherratt et al., 1999), which focus on the development of tumour heterogeneities as a result of tumour cell and macrophage interactions. Moreover, Webb et al. (2002) is concerned with receptor-ligand (Fas-FasL) dynamics, Kelly et al. (2002) investigate the process of macrophage infiltration into avascular tumours, Matzavinos et al. (2004), Matzavinos \& Chaplain (2004) and Chaplain \& Matzavinos (2006) focus on the dynamics of cytotoxic T cell-tumour cell interactions, and finally Foryś (2002) and Szymańska (2003) analyze various immune system and immunotherapy models in the context of cancer dynamics.

In this paper we develop a new mathematical model of immunotherapy, focusing on the role of antigen presentation and costimulatory signaling pathways in cancer immunology. We investigate the effect of different cancer vaccination protocols on the well-documented phenomena of cancer dormancy and recurrence, and we provide a possible explanation of why adoptive immunotherapy protocols can sometimes promote tumor growth instead of inhibiting it ${ }^{2}$ (Zhang et al., 2007), as opposed to active vaccination protocols based on tumor-antigen pulsed dendritic cells (Banchereau \& Palucka, 2005).

\footnotetext{
${ }^{2} \mathrm{~A}$ phenomenon that is usually referred to as immunostimulation.
}

\section{The Mathematical Model}

\subsection{Model formulation}

Let us consider a simplified process of a small, growing, avascular tumour which elicits a response from the host immune system and attracts a population of lymphocytes and antigen presenting cells (APCs). The growing tumour is directly attacked by cytotoxic T-lymphocytes (CTLs), which in the presence of tumour antigens undergo enhanced proliferation. Antigen presenting cells, such as dendritic cells or macrophages, internalize tumour cells through either phagocytosis or endocytosis, and display selected tumour antigenic peptides to the effector cells, i.e. the CTLs. In the following we only consider the class I MHC pathway of antigen presentation (Abbas et al., 2007), where the antigenic peptides are presented to and activate CTLs directly (as opposed to the class II MHC pathway that converges to the activation of helper T-cells that, in turn, activate the effector components of the host immune system).

The role of antigen presenting cells in the model is three-fold: (a) they internalize tumour cells and present tumour antigens to the effector cells, (b) they kill the internalized tumour cells through the action of proteolytic enzymes, and (c) they are subjected to programmed cell death as a result of their presenting of antigenic peptides to CTLs. The main effector functions of this system are performed by CTLs, which detect antigenic peptides either directly on tumour cells or through the presentation mechanism implemented by antigen presenting cells. Upon antigen recognition, CTLs bind to the target cell (which can be either a tumour cell or an antigen presenting cell with an internalized tumour cell). This binding event leads to the clustering of a large number of CTL receptors, trigerring a cascade of events that converge to the delivery of an apoptotic signal and the killing of the target cell.

The kinetic interactions between the various cell types of the model are described by the kinetic scheme given in Fig. 11. The model consists of ten time-dependent variables representing the total numbers of effector cells $E$, tumour cells $T$, naive antigen presenting cells $A$, tumour cell-loaded APCs $L$, CTL-tumour cell complexes $C_{T}$, CTL-APC complexes $C_{A}$, CTL-CTL complexes $C_{E}$, inactivated 


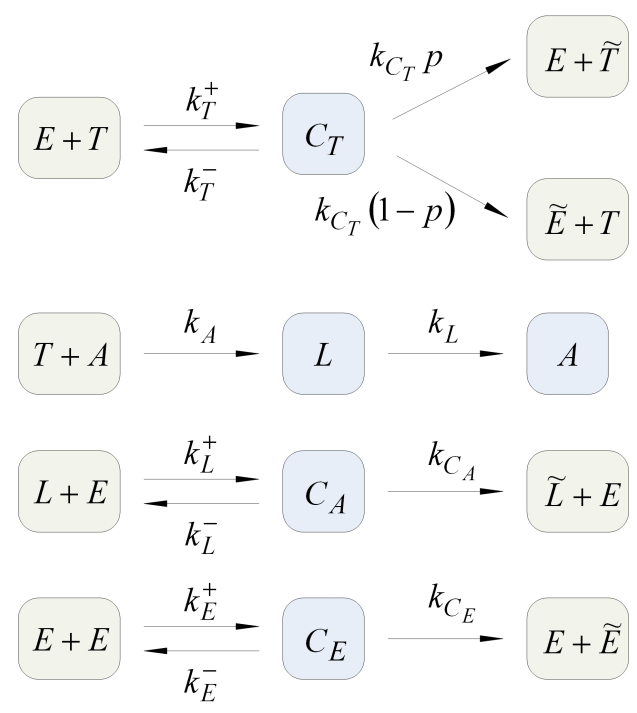

Figure 1: Schematic diagram of the interactions between effector cells (CTLs), tumour cells and antigen presenting cells.

CTLs $\widetilde{E}$, lethally-hit (or programmed-for-lysis) tumour cells $\widetilde{T}$ and programmed-for-lysis loaded APCs $\widetilde{L}$.

The parameters $k_{T}^{ \pm}, k_{C_{T}}, k_{A}, k_{L}, k_{L}^{ \pm}, k_{C_{A}}, k_{E}^{ \pm}$and $k_{C_{E}}$ are non-negative kinetic constants. Parameters $k_{T}^{+}$and $k_{T}^{-}$describe the rate of binding of CTLs to tumour cells and detachment of CTLs from tumour cells without damaging cells; $k_{C_{T}}$ is the rate of detachment of CTLs from tumour cells, resulting in an irreversible programming of the tumour cells for lysis (i.e. death) with probability $p$ or inactivating/killing CTLs with probability $(1-p)$. Similarly, $k_{L}^{ \pm}$and $k_{E}^{ \pm}$ are the corresponding kinetic constants for binding and detachment of CTLs to tumour cell-loaded antigen presenting cells and other CTLs without damaging cells. Parameters $k_{C_{A}}$ and $k_{C_{E}}$ are the rate constants for the CTL-mediated killing of (a) loaded antigen presenting cells and (b) CTLs respectively, whereas $k_{A}$ and $k_{L}$ characterize the rate of tumour cell internalization by antigen presenting cells and the destruction/lysis of internalized tumour cells.

The kinetic step that corresponds to the constant $k_{C_{T}}(1-p)$ models a direct "counterattack" of the tumour cells against effector immune cells. O'Connell et al. (1999) have shown that such a mechanism might be realized through the Fas receptor (Fas, Apo-1/CD95) and its ligand (FasL, CD95L). En- gagement of Fas on a target cell by FasL triggers a cascade of cellular events that result in programmedcell-death. Both these transmembrane proteins (belonging to the tumour necrosis factor (TNF) family of receptors and ligands) are expressed on the surface of immune cells, including T-lymphocytes and NK-cells. However, many non-lymphoid tumour cells also express FasL which can counterattack and kill the Fas-sensitive CTLs. On the other hand, most cancer cells, unlike normal cells, are relatively resistent to Fas-mediated apoptosis by the immune cells. Resistance to programmed-cell-death (apoptosis) through the Fas receptor pathway coupled with expression of the Fas ligand might enable many cancer cells to deliver a "counterattack" against attached cytotoxic lymphocytes.

In the formulation of the model, and in addition to the kinetic mechanisms described in Fig. 1, we consider other kinetic interaction terms accounting for the host immune system homeostasis, the enhanced proliferation of lymphocytes in the presence of antigen, etc.

\section{Cytotoxic T-lymphocytes}

We assume that there is a source term modelling the underlying lymphocyte production by the host immune system, a linear decay (death) term and an additional CTL proliferation term in response to the presence of the tumour cells. Combining these assumptions with the kinetics derived from Fig. 1 we have the following ordinary differential equation for CTLs:

$$
\begin{aligned}
\frac{d E}{d t}= & \overbrace{s_{1}}^{\text {supply }}-\overbrace{d_{1} E}^{\text {linear decay }}+\overbrace{\frac{f\left(C_{T}+C_{A}\right)}{g+T}}^{\text {proliferation }} \\
& -\overbrace{k_{T}^{+} E T+\left(k_{T}^{-}+k_{C_{T}} p\right) C_{T}-k_{L}^{+} L E}^{\text {mass action kinetics, according to Fig. 1 }} \\
& +\overbrace{k_{L}^{-} C_{A}+k_{C_{A}} C_{A}+2 k_{E}^{-} C_{E}}^{\text {mass action kinetics }} \\
& -\overbrace{2 k_{E}^{+} E^{2}+k_{C_{E} C_{E}}}^{\text {Fas/FasL interactions }},
\end{aligned}
$$

where $s_{1}, d_{1}, f, g, k_{E}^{ \pm}, k_{T}^{ \pm}, k_{L}^{ \pm}, k_{C_{E}}, k_{C_{T}}, k_{C_{A}}$ are all positive constants. The parameter $s_{1}$ represents the 'normal' rate of flow of mature lymphocytes into 
the tissue (non-enhanced by the presence of tumour cells).

The proliferation term $f\left(C_{T}+C_{A}\right) /(g+T)$ represents the experimentally observed enhanced proliferation of CTLs in response to the tumour. Similar functional forms for this term have been derived through data fitting and used by Kuznetsov et al. (1994), Chaplain et al. (1998) and Matzavinos et al. (2004). This functional form is also consistent with a model in which one assumes that the enhanced proliferation of CTLs is due to signals, such as released interleukins, generated by effector cells in target cell-CTL complexes (where a target cell is either a tumour cell or a antigen presenting cell with an internalized tumour cell). We note that the growth factors that are secreted by lymphocytes in complexes (e.g. IL-2) act mainly in an autocrine fashion. That is to say they act on the cell from which they have been secreted. We assume that the growth factors are produced when lymphocytes are activated by target cell-CTL interactions. Thus we define effector cell proliferation to be proportional to target cell-CTL complex density $C_{T}+C_{A}$. The kinetic interaction terms related to the rate constants $k_{E}^{+}, k_{E}^{-}$and $k_{C_{E}}$ correspond to Fas/FasL interactions between effector cells (Marsden \& Strasser, 2003).

\section{Tumour cells}

The growth dynamics of pre-angiogenic tumours, in the absence of an immune system response, may be described adequately by the logistic equation:

$$
\frac{d T}{d t}=b_{1} T\left(1-b_{2} T\right)
$$

which takes into account a density limitation of growth (Prigogine \& Lefever, 1980; Durand \& Sutherland, 1984). The maximal growth rate of the tumour cell population is $b_{1}$, which incorporates both cell multiplication (mitosis) and death, and the maximum density of the tumour cells is represented by the parameter $b_{2}^{-1}$.

An alternative approach is to modify the logistic growth kinetics by incorporating terms modelling competition for space between various cell types (Gatenby, 1995; Gatenby, 1996). However, in the framework of our model, we will assume that the CTLs do not compete with the tumour cells for space. This is a reasonable assumption since according to observations (Kyle et al., 1999) the volume of extracellular space in tumours is typically in the range $25-65 \%$ of the total volume of cells and hence there is enough space for the migration of lymphocytes within a tumour. Also, tumour cells lack the contact inhibition properties of normal cells and destroy the extracellular matrix. This allows the lymphocytes to migrate into the tumour tissue faster than in normal tissue, which has regular extracellular matrix. Therefore we do not explicitly include a term for space competition between the tumour cells and the lymphocytes and thus a logistic growth term is, we believe, a good first modelling approximation to the tumour growth kinetics.

In the presence of CTLs and antigen presenting cells, the ODE governing the tumour growth dynamics in conjunction with the interactions dictated by the kinetic scheme in Fig. 1 is:

$$
\begin{aligned}
\frac{d T}{d t}= & -\overbrace{k_{T}^{+} E T+\left(k_{T}^{-}+k_{C_{T}}(1-p)\right) C_{T}}^{\text {mass action kinetics, according to Fig. 1 }} \\
& -\overbrace{k_{A} T A}^{\text {mass action kinetics }}+\overbrace{b_{1} T\left(1-b_{2} T\right)}^{\text {logistic growth }}
\end{aligned}
$$

where $b_{1}, b_{2}, p, k_{T}^{ \pm}, k_{C_{T}}$ and $k_{A}$ are positive parameters.

\section{Antigen presenting cells}

The antigen presenting cell population is represented by two variables, distinguishing between those antigen presenting cells that are associated with an internalized tumour cell and those that are not. The former population is denoted by $L$ (loaded cell population), whereas the latter is denoted by $A$.

As in the case of CTLs, the model incorporates a source term modelling the underlying cell production by the host immune system and a linear decay (death) term. Combining these processes with the kinetic scheme in Fig. 1, we get the following equation for the non-tumour-bearing population:

$$
\frac{d A}{d t}=\overbrace{s_{2}}^{\text {supply }}-\overbrace{d_{2} A}^{\text {linear decay }}-\overbrace{k_{A} T A+k_{L} L}^{\text {mass action kinetics }}
$$

where the parameters $s_{2}$ and $d_{2}$ correspond to the flow of antigen presenting cells into the tissue and the decay constant due to cell death respectively. 
Table 1: Estimated kinetic rate constants.

\begin{tabular}{|l|l||l|l||l|l|}
\hline Symbol & Value & Symbol & Value & Symbol & Value \\
\hline \hline$g$ & $2.02 \times 10^{7}$ cells & $b_{2}$ & $2.0 \times 10^{-9}$ cells $^{-1}$ & $k_{T}^{-}$ & 24 day $^{-1}$ \\
$k_{T}^{+}$ & $1.3 \times 10^{-7}$ day $^{-1}$ cells $^{-1}$ & $k_{C_{T}}$ & 7.2 day $^{-1}$ & $p$ & 0.9997 \\
$d_{1}$ & 0.0412 day $^{-1}$ & $f$ & $0.2988 \times 10^{8}$ day $^{-1}$ cells $^{-1}$ & $b_{1}$ & 0.18 day $^{-1}$ \\
$s_{1}$ & $1.36 \times 10^{4}$ day $^{-1}$ cells & $k_{A}$ & $0.5 \times 10^{-6}$ day $^{-1}$ cells $^{-1}$ & $k_{L}$ & 10.0 day $^{-1}$ \\
$k_{L}^{+}$ & $1.3 \times 10^{-7}$ day $^{-1}$ cells $^{-1}$ & $k_{L}^{-}$ & 24 day $^{-1}$ & $k_{C_{A}}$ & 7.2 day $^{-1}$ \\
$k_{E}^{+}$ & $1.3 \times 10^{-7}$ day $^{-1}$ cells $^{-1}$ & $k_{E}^{-}$ & 24 day $^{-1}$ & $k_{C_{E}}$ & 7.2 day $^{-1}$ \\
$s_{2}$ & $1.36 \times 10^{4}$ day $^{-1}$ cells & $d_{2}$ & 0.0412 day $^{-1}$ & & \\
\hline
\end{tabular}

The dynamics of the loaded cell population are governed by the kinetic scheme in Fig. 1, and the corresponding equation for $L$ is:

$$
\frac{d L}{d t}=k_{A} T A-k_{L} L-k_{L}^{+} L E+k_{L}^{-} C_{A}
$$

\section{Cell complexes}

The dynamics of cell-complexes are governed by the kinetics derived from Fig. 1. Therefore the equations for the complexes are given by:

$$
\begin{aligned}
\frac{d C_{T}}{d t} & =k_{T}^{+} E T-\left(k_{T}^{-}+k_{C_{T}}\right) C_{T}, \\
\frac{d C_{A}}{d t} & =k_{L}^{+} L E-\left(k_{L}^{-}+k_{C_{A}}\right) C_{A}, \\
\frac{d C_{E}}{d t} & =k_{E}^{+} E^{2}-\left(k_{E}^{-}+k_{C_{E}}\right) C_{E} .
\end{aligned}
$$

\section{Cells programmed for lysis}

The time-dependent variables corresponding to the tumour cells and APCs that have been programmed for lysis are "slave variables" of the system and don't provide any feedback to the equations for the other cell types. Hence, in the following we focus on the system of equations (1)-(8).

\subsection{Estimation of parameters}

In order to carry out an analysis of the model by numerical methods it is useful to estimate values for the parameters obtained from experimental data and work with a non-dimensionalized system of equations.

The murine $\mathrm{B}$ cell lymphoma $\left(\mathrm{BCL}_{1}\right)$ is used as an experimental model of tumour dormancy in mouse
(Siu et al., 1986; Uhr \& Marches, 2001). It has been demonstrated that $\mathrm{CD}^{+}$T-cells (CTLs) are required for inducing and maintaining dormancy in $\mathrm{BCL}_{1}$. In these experiments $\mathrm{CD} 8^{+} \mathrm{T}$ cells are enhanced with anti-Id antibodies into inducing dormancy by secreting INF- $\gamma$. A description of the growth kinetics of a $\mathrm{BCL}_{1}$ lymphoma in the spleen of recipient mice, chimeric with respect to the Major Histocompatibility Complex (Siu et al., 1986), was provided by the model of Matzavinos et al. (2004).

The kinetic constants that correspond to the interactions of CTLs with tumour cells in the model developed in this paper have been obtained from Matzavinos et al. (2004). The remaining of the kinetic constants have been chosen on the basis of generic order of magnitude estimates. The latter were obtained by comparison of the associated time scales with those corresponding to the fitted kinetic constants of Matzavinos et al. (2004). Table 1 shows the values of the kinetic constants used in the numerical simulations of the following section.

The system of equations (1)-(8) is closed by applying appropriate initial conditions. The initial cell counts of effector and tumour cells are those used by Matzavinos et al. (2004) and are given by:

$$
E_{0}=\frac{s_{1}}{d_{1}} \quad \text { and } \quad T_{0}=\frac{1}{b_{2}} .
$$

Under physiological conditions, in the absence of a tumour and assuming that the Fas/FasL apoptotic pathway is inactive, the steady-state value of $E$ is $s_{1} / d_{1}$ and therefore this is the value we have taken for the initial cell count $E_{0}$. Similarly, in the absence of an immune response,the steady-state tumour cell count is $1 / b_{2}$ and this is what we take as the initial 
(a) Tumour recurrence under different initial APC counts

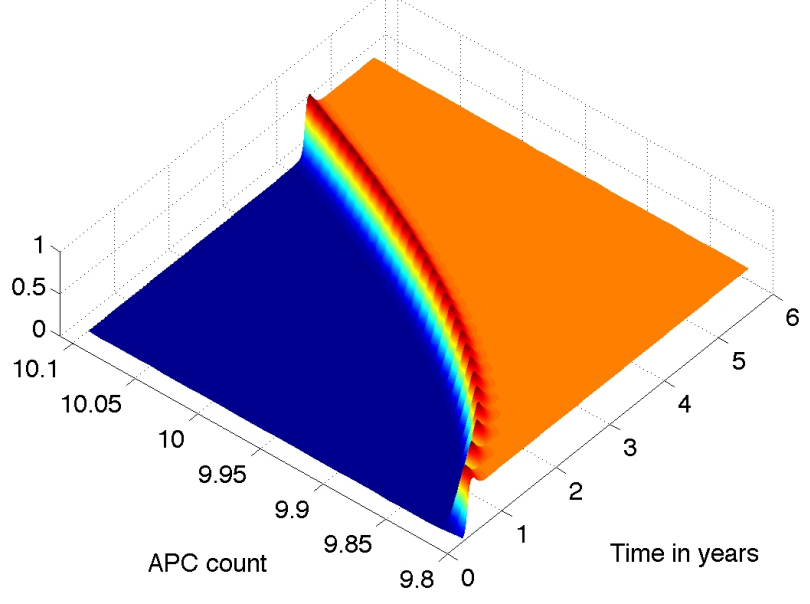

(b) Tumour recurrence under different initial CTL counts

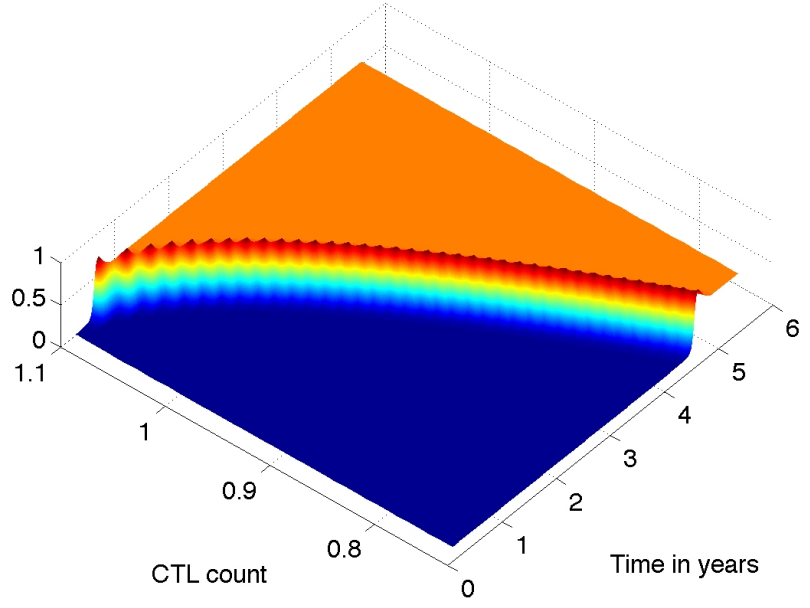

Figure 2: Evolution in time of (non-dimensionalized) tumour cell counts under different initial numbers of (a) antigen presenting cells and (b) cytotoxic Tlymphocytes.

tumour cell count $T_{0}$. It is assumed that there are no cell complexes or tumour cell-loaded APCs initially. Finally, the initial CTL to APC ratio is assumed to be $1: 103$

The closed system has been non-dimensionalized by choosing order-of-magnitude scales according to the initial conditions, and the numerical results in the next section are given in terms of the nondimensionalized system.

\footnotetext{
${ }^{3}$ Estimates of the CTL to APC ratio in the literature vary from $10: 1$ to $1: 30$ (in tumour infiltrates).
}

\section{Results}

The non-dimensionalized system was solved numerically under different experimental settings using the stiff solver of the XPP numerical package (Ermentrout, 2002). A stiff solver is needed for solving numerically the ODE model developed in this paper due to the wide range of parameter values in Table 1 .

The main focus of the numerical experiments was: (a) to investigate the relative importance of CTLs and antigen presenting cells on tumour dormancy and tumour recurrence and (b) to quantify the effectiveness of different cancer vaccination protocols. The vaccination protocols considered were based on the administration of antigen presenting cell vaccines (Banchereau \& Palucka, 2005), and the effectiveness of the latter was compared to that of an adoptive (passive) immunotherapy approach based on the administration of CTLs (Gattinoni et al., 2006). An excellent review of the current cancer immunotherapy approaches is given by Gilboa (2004).

Figures 2(a) and 2(b) show the results of a computational experiment that quantifies how different numbers of CTLs and antigen presenting cells affect the time period between tumour regression and recurrence. All parameter values for these simulations were set according to Section 2.2. The initial conditions for variables $A$ in Fig. 2(a) and $E$ in Fig. 2(b) were varied in a biologically-relevant range around the values adopted in Section 2.2 .

For all parameter values investigated, in both Figs. 2(a) and 2(b), the tumour cell population intially decreases in number before subsequently settling to some stationary value for a finite period of time. For visualization purposes, the transient decrease in the number of tumour cells is not shown. The reduced tumour bulk attained in the simulations after tumour regression persists until the tumour recurs at a time that depends on the initial CTL and antigen presenting cell counts. As shown in Fig. 2(a), the initial antigen presenting cell count correlates well with the tumour dormancy period, and the model suggests that elevated numbers of antigen presenting cells result in significantly delaying tumour recurrence. These results also suggest a potential role for antigen presenting cells as biological markers, indicating high-risk time periods for tumour recurrence. 
(a) Effect of parameter $\mathrm{k}_{\mathrm{C}_{\mathrm{E}}}$ on tumour recurrence

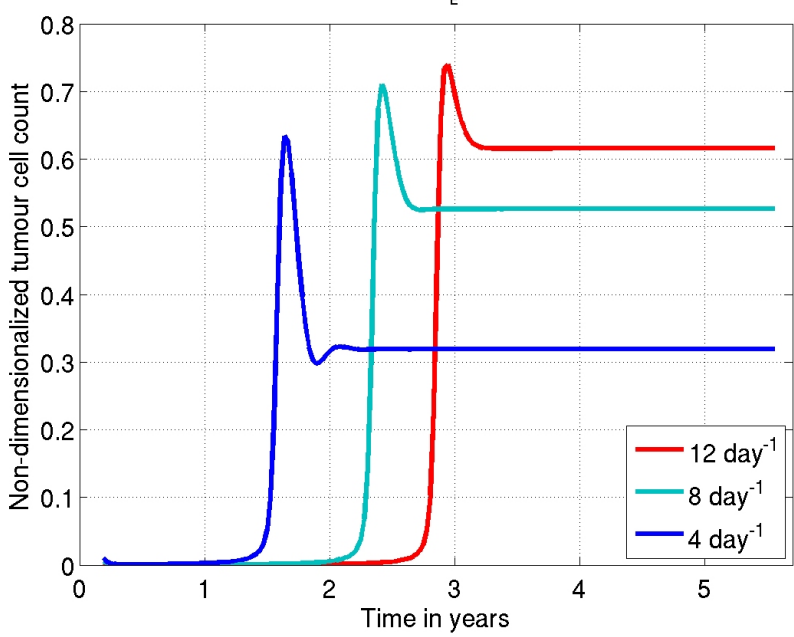

(b) Continuation of the solution with respect to $\mathrm{K}_{\mathrm{C}_{\mathrm{E}}}$

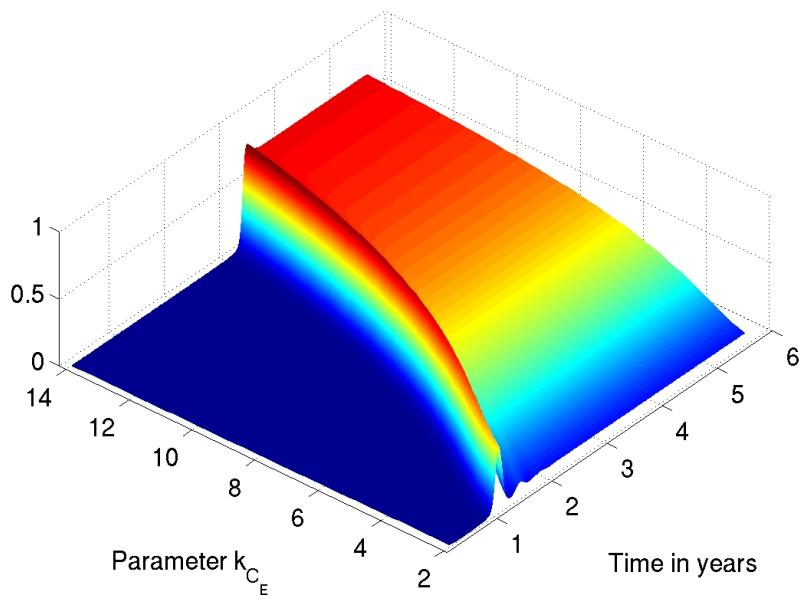

Figure 3: (a) Effect of parameter $k_{C_{E}}$ on the dormancy period and the recurrence of the disease. (b) Continuation of the non-dimensionalized solution for variable $T$ with respect to $k_{C_{E}}$.

Figure 2(b) shows an interesting, counter-intuitive phenomenon. According to the model under investigation, elevated initial numbers of CTLs (as compared to the base value in Section 2.2) result in a reduced period of cancer dormancy and an early recurrence of the disease. Moreover, relatively reduced initial numbers of CTLs result in a prolonged dormancy period. This phenomenon should be attributed to the cytotoxicity of activated lymphocytes to each other (Fas/FasL interactions). Indeed, initial conditions that are characterized by elevated num- bers of CTLs result not only to tumour cell killing, but also to CTL killing, enabling tumour cells to escape cancer dormancy on a faster time scale.

These results offer a possible explanation for a number of reported failures of CTL vaccinebased therapeutic approaches to certain carcinomas (see, for example, Zhang et al., 2007). The rationale of such approaches is based on the welldocumented correlation between melanoma patient survival and tumour infiltrating lymphocyte (TIL) counts (Weinberg, 2007). However, TIL populations are highly heterogeneous, including among others CTLs (CD8+ cells), natural killer-like (NKlike) cells and/or lymphokine activated killer (LAK) cells, with different TIL subpopulations showing different degrees of sensitivity to Fas/FasL mediated apoptosis. Hence, the reported correlation between melanoma patient survival and TIL counts does not contradict the results in this section, since the latter are related to CTLs that are responsive to the Fas/FasL signalling pathway. Moreover, the dynamics of the model under investigation are consistent with well-documented tumour recurrence phenomena, following the application of CTL-based vaccination protocols (Zhang et al., 2007).

In addition to observing the effect of different cell counts on the dormancy period, we also investigated how the latter is affected by the time scale on which lymphocytes detect and bind to tumour cells, as well as by the cytotoxicity of CTLs to each other. Figure 3(a) shows the evolution in time of the tumour cell population for three different values of the kinetic parameter $k_{C_{E}}$ that measures the rate by which CTLs inactivate/kill each other through Fas/FasL interactions. Interestingly enough, reducing parameter $k_{C_{E}}$ leads to early tumour recurrence associated with a reduced tumour bulk. In contrast, increasing $k_{C_{E}}$ results in prolonging the dormancy period and delaying the recurrunce of the disease. However, the model predicts that increasing $k_{C_{E}}$ also results in an enlarged tumour bulk after recurrence. Figure 3(b) shows a continuation of the (non-dimensionalized) solution for variable $T$ with respect to parameter $k_{C_{E}}$.

Interesingly enough, reducing parameter $k_{C_{E}}$ below the value of 1 day $^{-1}$ does not result in complete eradication of the tumour mass, as one would ex- 


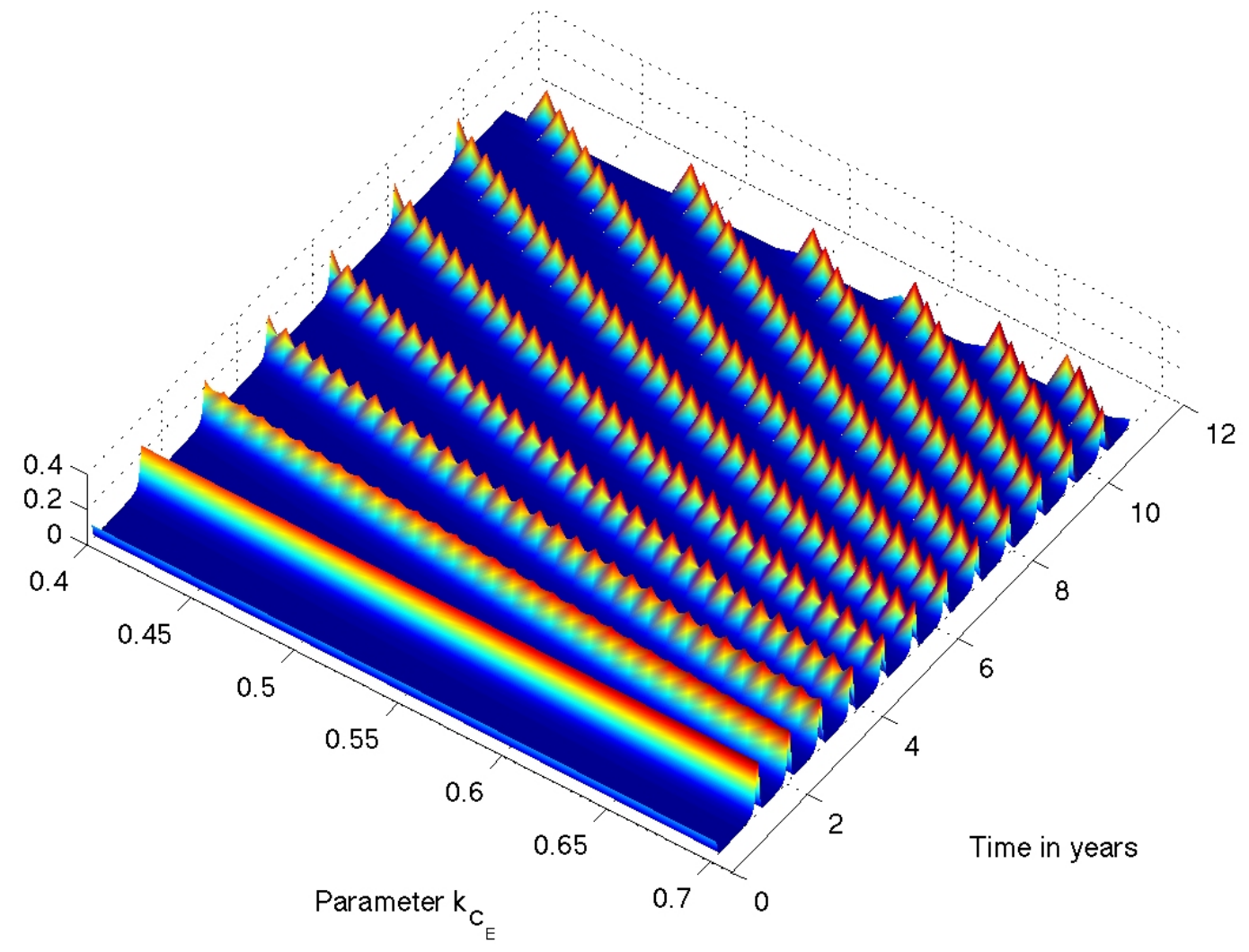

Figure 4: Emergence of periodic recurrences for small values of parameter $k_{C_{E}}$

pect by extrapolation of the data in Fig. 3(b). Instead, in this parameter regime, the evolution of the disease is characterized by periodic recurrences; a phenomenon that frequently appears in the context of general immune-pathogen interactions (Wodarz, 2007) and has also been documented in the specific case of tumour-immune interactions (Kirschner \& Panetta, 1998; Kuznetsov et al., 1994; Matzavinos \& Chaplain, 2004). Figure 4 shows the dependence of the emerging oscillatory solutions on parameter $k_{C_{E}}$. The model predicts that, although the first recurrence of the disease is independent of the value of $k_{C_{E}}$, subsequent recurrences will strongly depend on the sensitivity of the effector cells to Fas/FasL mediated apoptosis. Numerical continuation of the system with the XPP implementation of the AUTO continuation software (Ermentrout, 2002) confirmed that these oscillatory dynamics emerge through a
Hopf bifurcation with respect to parameter $k_{C_{E}}$.

Figure 5(a) shows the evolution in time of the tumour cell population for four different values of the kinetic parameter $k_{T}^{+}$, which determines the time scale of CTL-binding to tumour cells. As can be seen, elevated values for $k_{T}^{+}$result in both a prolonged dormancy period and a reduced tumour bulk after recurrence of the disease. This result indicates the importance of identifying immunodominant tumour peptides in the development of cancer vaccines (Raitakari et al., 2003) and suggests a cellular process, the facilitation of which would enhance the effectiveness of the vaccine.

In addition to vaccine effectiveness, we investigated computationally the effectiveness of different vaccine administration protocols. Figure 5 (b) shows how the time period elapsed before recurrence of the disease depends on the number of monthly im- 

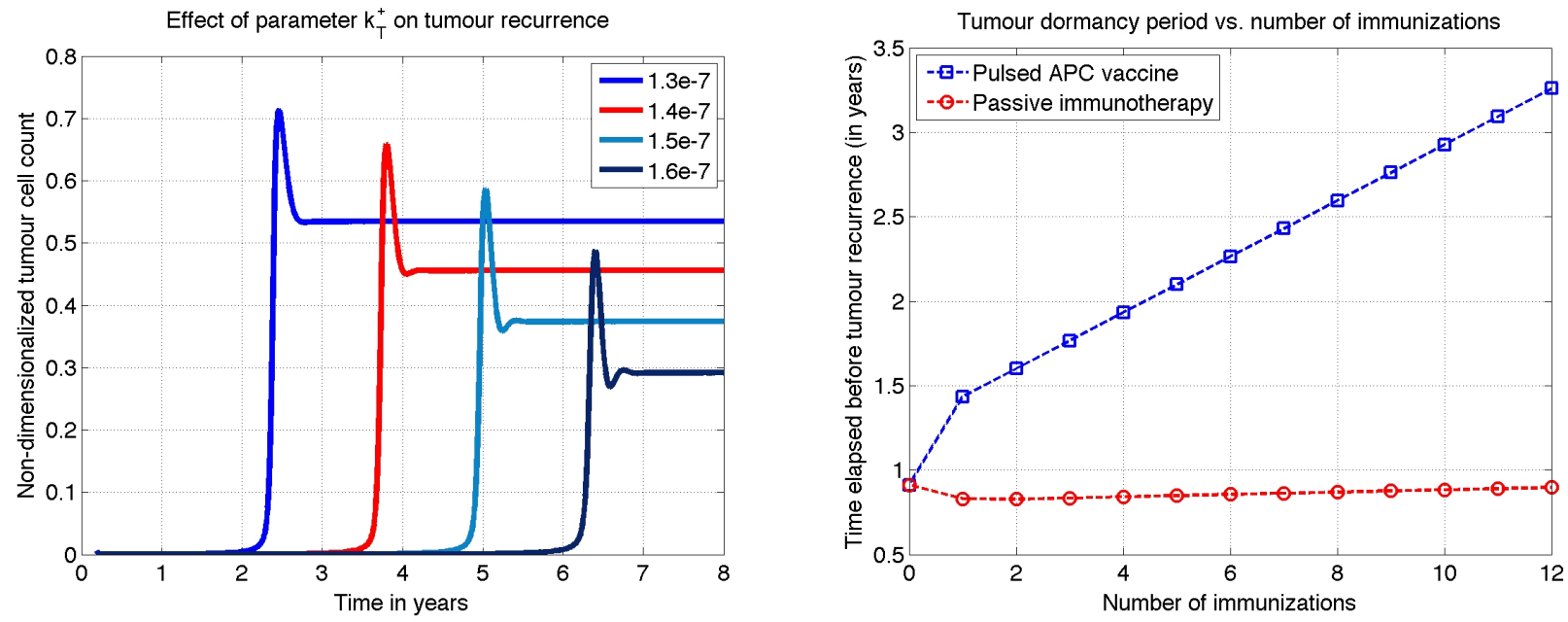

Figure 5: (a) Effect of parameter $k_{T}^{+}$on the dormancy period and the recurrence of the disease, (b) Plot of the tumour dormancy time period versus the number of immunizations for two different cancer vaccination protocols.

munizations under two different vaccination strategies. As can be seen active vaccination with tumourantigen pulsed APCs is associated with a positive correlation between the number of immunizations and the tumour dormancy period, and it is generally more effective than adoptive immunotherapy protocols. In contrast, the adoptive (passive) imnmunotherapy protocol simulated (based on CTL vaccines) fails to mount an effective immune response even in the case of a significant number of repeated immunizations.

\section{Discussion}

In this paper we have developed a mathematical model to describe the growth dynamics of an immunogenic tumour in the presence of an active immune response. In particular, we focused attention upon the interaction of tumour cells with $\mathrm{CD} 8^{+}$cytotoxic T-lymphocytes and professional antigen presenting cells in a relatively small, multicellular tumour, without central necrosis and at some stage prior to tumour-induced angiogenesis (Weinberg, 2007). Following the approach of Matzavinos et al. (2004) and Matzavinos \& Chaplain (2004), the cytotoxic T-lymphocytes were assumed to interact with the tumour cells in such a way that lymphocyte- tumour cell complexes were formed. These complexes resulted in either the death of the tumour cells (the normal situation) or the inactivation (sometimes even the death) of the lymphocytes.

The model developed in this paper extends the work of Chaplain \& Matzavinos (2006) by investigating the role of antigen presentation and costimulatory signaling pathways on the well-documented phenomena of cancer dormancy and recurrence. In particular, in the formulation of the model we considered a generic type of professional antigen presenting cell that was assumed to internalize tumour cells through either phagocytosis or endocytosis and display selected tumour antigenic peptides to the effector cells, i.e., the cytotoxic T-lymphocytes (Abbas et al., 2007).

The dynamics of the model were investigated by means of numerical simulations, and a number of interesting, counter-intuitive phenomena were discovered. It was demonstrated that, under the assumptions of the model, adoptive immunotherapy protocols have the potential to promote tumour growth instead of inhibiting it. These results are consistent with well-documented tumour recurrence phenomena following the application of CTL-based vaccination protocols (Zhang et al., 2007). In contrast, active vaccination with tumour-antigen pulsed APCs (Banchereau \& Palucka, 2005) was shown to be gen- 
erally more effective than adoptive immunotherapy protocols in inhibiting tumour growth and recurrence in the model under investigation.

The numerical predictions of our model offer a possible explanation for the uncontrolled behaviour of a number of lymphocyte vaccine-based therapeutic approaches to certain carcinomas, and our modelling and analysis offers the potential for quantitative analysis of mechanisms of tumour-cell-host-cell interactions and for the optimization of immunotherapy and genetically engineered anti-tumour vaccines.

\section{Acknowledgements}

The authors would like to thank Avner Friedman for advice and encouragement. This material is based upon work supported by the National Science Foundation under agreement No. 0635561.

\section{References}

Abbas, A., Lichtman, A. \& Pillai, S. (2007), Cellular and Molecular Immunology, 6th edn, Saunders/Elsevier.

Ambrosi, D., Bellomo, N. \& Preziosi, L. (2002), 'Modelling tumor progression, heterogeneity, and immune competition', J. Theor. Medicine 4, 51-65.

Arlotti, L., Gamba, A. \& Lachowicz, M. (2002), 'A kinetic model of tumor/immune system cellular interactions', J. Theor. Medicine 4, 39-50.

Banchereau, J. \& Palucka, A. (2005), 'Dendritic cells as therapeutic vaccines against cancer', Nature Reviews Immunology 5, 296-306.

Bellomo, N. \& Preziosi, L. (2000), 'Modelling and mathematical problems related to tumor evolution and its interaction with the immune system', Math. Comp. Modelling 32, 413-452.

Bellomo, N., Bellouquid, A. \& De Angelis, E. (2003), 'The modelling of the immune competition by generalized kinetic (Boltzmann) models: Review and research perspectives', Math. Comp. Modelling 37, 65-86.
Bellomo, N., Firmani, B. \& Guerri, L. (1999), 'Bifurcation analysis for a nonlinear system of integro-differential equations modelling tumorimmune cells competition', Appl. Math. Letters 12, 39-44.

Chaplain, M. \& Matzavinos, A. (2006), Mathematical modelling of spatio-temporal phenomena in tumour immunology, in A. Friedman, ed., 'Tutorials in Mathematical Biosciences III: Cell Cycle, Proliferation, and Cancer', Vol. 1872 of Lecture Notes in Mathematics, Springer, pp. 131-183.

Chaplain, M., Kuznetsov, V., James, Z. \& Stepanova, L. (1998), Spatio-temporal dynamics of the immune system response to cancer, in M. A. Horn, G. Simonett \& G. Webb, eds, 'Mathematical Models in Medical and Health Sciences', Vanderbilt University Press, pp. 120 .

De Angelis, E., Delitala, M., Marasco, A. \& Romano, A. (2003), 'Bifurcation analysis for a mean field modelling of tumor and immune system competition', Math. Comp. Modelling 37, 1131-1142.

Durand, R. \& Sutherland, R. (1984), 'Growth and cellular characteristics of multicell spheroids', Recent Results in Cancer Research 95, 24-49.

Ermentrout, G. (2002), Simulating, Analyzing, and Animating Dynamical Systems: A Guide to XPPAUT for Researchers and Students, Vol. 14 of Software, Environments, and Tools, SIAM.

Foryś, U. (2002), 'Marchuk's model of immune system dynamics with application to tumour growth', J. Theor. Medicine 4, 85-93.

Gatenby, R. (1995), 'Models of tumor-host intearcation as competing populations: Implications for tumor biology and treatment', J. theor. Biol. 176, 447-455.

Gatenby, R. (1996), 'Application of competition theory to tumour growth: Implications for tumour biology and treatment', European Journal of Cancer 32A, 722-726. 
Gattinoni, L., Jr., D. P., Rosenberg, S. \& Restifo, N. (2006), 'Adoptive immunotherapy for cancer: building on success', Nature Reviews Immunology 6, 383-393.

Gilboa, E. (2004), 'The promise of cancer vaccines', Nature Reviews Cancer 4, 401-411.

Kelly, C., Leek, R., Byrne, H., Cox, S., Harris, A. \& Lewis, C. (2002), 'Modelling macrophage infiltration into avascular tumours', J. Theor. Med. 4, 21-38.

Kirschner, D. \& Panetta, J. (1998), 'Modeling immunotherapy of the tumor immune interaction', J. Math. Biol. 37, 235-252.

Kuznetsov, V., Makalkin, I., Taylor, M. \& Perelson, A. (1994), 'Nonlinear dynamics of immunogenic tumours: Parameter estimation and global bifurcation analysis', Bull. Math. Biol. 56, 295321.

Kyle, A., Chan, C. \& Minchinton, A. (1999), 'Characterization of three-dimensional tissue cultures using electrical impedance spectroscopy', Biophys J. 76(5), 2640-2648.

Marsden, V. \& Strasser, A. (2003), 'Control of apoptosis in the immune system: Bcl-2, BH3only proteins and more', Annu. Rev. Immunol. 21, 71-105.

Matzavinos, A. \& Chaplain, M. (2004), 'Travellingwave analysis of a model of the immune response to cancer', C.R. Biologies 327, 9951008 .

Matzavinos, A., Chaplain, M. \& Kuznetsov, V. (2004), 'Mathematical modelling of the spatiotemporal response of cytotoxic T-lymphocytes to a solid tumour', Mathematical Medicine and Biology: A Journal of the IMA 21, 1-34.

O'Connell, J., Bennett, M., O'Sullivan, G., Collins, J. \& Shanahan, F. (1999), 'The Fas counterattack: cancer as a site of immune privilege', Immunol. Today 20(1), 46-50.

Owen, M. \& Sherratt, J. (1997), 'Pattern formation and spatio-temporal irregularity in a model for macrophage-tumour interactions', J. Theor. Biol. 189, 63-80.

Owen, M. \& Sherratt, J. (1998), 'Modelling the macrophage invasion of tumours: Effects on growth and composition', IMA J. Math. Appl. Med. Biol. 15, 165-185.

Owen, M. \& Sherratt, J. (1999), 'Mathematical modelling of macrophage dynamics in tumours', Math. Models Meth. Appl. Sci. 9, 513-539.

Parmiani, G. \& Lotze, M., eds (2002), Tumor Immunology: Molecularly Defined Antigens and Clinical Applications, Vol. 1 of Tumor Immunology and Immunotherapy Series, Taylor \& Francis.

Prigogine, I. \& Lefever, R. (1980), 'Stability problems in cancer growth and nucleation', Comp. Biochem. Physiol. 67, 389-393.

Raitakari, M., Brown, R., Gibson, J. \& Joshua, D. (2003), 'T cells in myeloma', Hematological Oncology 21(1), 33-42.

Ries, L., Melbert, D., Krapcho, M., Mariotto, A., Miller, B., Feuer, E., Clegg, L., Horner, M., Howlader, N., Eisner, M., Reichman, M. \& Edwards, B., eds (2007), SEER Cancer Statistics Review, 1975-2004, National Cancer Institute. Bethesda, MD. http://seer.cancer.gov/csr/1975_2004/.

Sherratt, J., Perumpanani, A. \& Owen, M. (1999), Pattern formation in cancer, in M. Chaplain, G. Singh \& J. McLachlan, eds, 'On Growth and Form: Spatio-temporal Pattern Formation in Biology', John Wiley \& Sons Ltd.

Siu, H., Vitetta, E., May, R. \& Uhr, J. (1986), 'Tumour dormancy. regression of BCL tumour and induction of a dormant tumour state in mice chimeric at the major histocompatibility complex', J. Immunol. 137, 1376-1382.

Szymańska, Z. (2003), 'Analysis of immunotherapy models in the context of cancer dynamics', Appl. Math. Comp. Sci. 13, 407-418. 
Uhr, J. \& Marches, R. (2001), 'Dormancy in a model of murine B cell lymphoma', Seminars in Cancer Biology 11, 277-283.

Webb, S., Sherratt, J. \& Fish, R. (2002), 'Cells behaving badly: a theoretical model for the Fas/FasL system in tumour immunology', Mathematical Biosciences 179, 113-129.

Weinberg, R. (2007), The Biology of Cancer, Garland Science, Taylor and Francis Group.

Wodarz, D. (2007), Killer Cell Dynamics: Mathematical and Computational Approaches to Immunology, Vol. 32 of Interdisciplinary Applied Mathematics, Springer.

Zhang, X., Moche, J., Farber, D. \& Strome, S. (2007), 'Vaccine-based approaches to squamous cell carcinoma of the head and neck', Oral Diseases 13, 17-22. 